\title{
BMC Nephrology reviewer acknowledgement 2014
}

Hayley Henderson

\section{Contributing reviewers}

The editors of BMC Nephrology would like to thank all our reviewers who have contributed to the journal in Volume 15 (2014).

Kevin Abbott

USA

Samar Abd Elhafeez

Egypt

Khaled Abdel-Kader

USA

Matthew Abramowitz

USA

Matteo Accetturo

Italy

Teresa Adragao

Portugal

Varun Agrawal

USA

Sabah Alharazy

Malaysia

John Allen

Singapore

Charles Alpers

USA

Michele Andreucci

Italy

Elio Antonucci

Italy

Michel Aparicio

France

\author{
Marta Arazzi \\ Italy
}

Christos Argyropoulos

USA

Masashi Asanoma

Japan

Brad Astor

USA

Alice Atzeni

Italy

Vincent Audard

France

Ahmad Taher Azar

Egypt

Marcelo Bacci

Brazil

Sunil Badve

Australia

Jyoti Baharani

UK

Matthew Bailey

UK

Phillippa Bailey

UK

Daniel Balkovetz

USA
Tanushree Banerjee

USA

Dongsik Bang

Korea, South

Irina Barash

USA

Carlo Basile

Italy

Rommel Bataclan

Philippines

Andrea Bauer

Brazil

Sahar Bayat

France

Justin Belcher

USA

Antonio Bellasi

Italy

Iddo Ben-Dov

USA

Laureline Berthelot

France

Francois Berthoux

France

Sebastjan Bevc

Slovenia

Correspondence: Hayley.henderson@biomedcentral.com

BioMed Central, Floor 6, 236 Gray's Inn Road, London WC1X 8HB, UK 
Markus Bitzer

USA

Natasa Bogavac-Stanojevic

Serbia

Piergiorgio Bolasco

Italy

Jason Bonomo

USA

Annalisa Botta

Italy

Josée Bouchard

Canada

L. Ebony Boulware

USA

Amarpali Brar

USA

Lauren Bresee

Canada

Sergey Brodsky

USA

Godela Brosnahan

USA

Steven Brunelli

USA

Bjoern Buchholz

Germany

Emmanuel Burdmann

Brazil

Nilka Burrows

USA

Gianfranca Cabiddu

Italy

Ronit Calderon-Margalit

Israel

Ruth Campbell

USA

Bernard Canaud

France

Angel Candela

Spain

Pietro Canetta

USA

Riccardo Cao

Italy
Alessandro Capitanini

Italy

Ben Caplin

UK

Cristina Capusa

Romania

Thomas Carroll

USA

Andre Carvalho

Brazil

Francesco Gaetano Casino

Italy

Giuseppe Castellano

Italy

Davide Catucci

Italy

Alejandro Chade

USA

Alex Chang

USA

Anthony Chang

USA

Hung-Chun Chen

Taiwan

Nan Chen

China

Tianxin Chen

China

Ying Chen

USA

Wai Leng Chow

Singapore

Byung Ha Chung

Korea, South

Mariann Churchwell

USA

Franco Citterio

Italy

Naomi Clyne

Sweden

Patrick Coates

Australia

Steven Coca

USA
Arthur Cohen

USA

Eric Cohen

USA

Jordana Cohen

USA

Joakim Cordtz

Denmark

Tom Cornelis

Netherlands

Frank Costantini

USA

Cécile Couchoud

France

Adrian Covic

Romania

Daniel Coyne

USA

Adamasco Cupisti

Italy

Marica Cutajar

UK

Wendy Dahl

USA

Aaron Dall

USA

Andrew Davenport

UK

John Davison

UK

Marc De Broe

Belgium

Mark De Caestecker USA

Andreana De Mauri Italy

Agostino De Pascale Italy

Sophie De Seigneux

Switzerland

Anne-Emilie Decleves

Belgium

Claudia Del Corso

Italy 
Dorella Del Prete

Italy

Lucia Del Vecchio

Italy

Pierre Delanaye

Belgium

Asterios Deligiannis

Greece

Sevag Demirjian

USA

Claire Den Hoedt

Netherlands

Patrice Deteix

France

Prasad Devarajan

USA

Patrick D'Haese

Belgium

Biagio Raffaele Di Iorio

Italy

Luca Di Lullo

Italy

Antonello Di Paolo

Italy

Clarissa Diamantidis

USA

Jun-Young Do

Korea, South

Mirela Dobre

USA

Mark Dockrell

UK

Kent Doi

Japan

Sonia Doi

USA

Tevfik Ecder

Turkey

Justin B. Echouffo-Tcheugui

USA

Charles Edelstein

USA

Philipp Eller

Austria
Ziad El-Zoghby

USA

F. Fevzi Ersoy

Turkey

Ciro Esposito

Italy

Vittoria Esposito

Italy

Michelle Estrella

USA

Paul Evans

UK

Colomba Falcone

Italy

Christopher Farmer

UK

Robert Fassett

Australia

Claude Ferec

France

Mariano Feriani

Italy

Hafedh Fessi

France

Fredric O Finkelstein

USA

Patrik Finne

Finland

Jennifer Flythe

USA

Daniele Focosi

Italy

Donald Fraser

UK

Simon Fraser

UK

Robert Frithiof

Sweden

Masafumi Fukagawa

Japan

Martina Gaggl

Austria

Ranil Gajanayaka

USA
Andrea Galassi

Italy

Daniel Gale

UK

Neetika Garg

USA

Karim Gariani

Switzerland

Giacomo Garibotto

Italy

Seyed Mansour

Gatmiri Iran

Joseph Gaut

USA

Adam Gaweda

USA

Duvuru Geetha

USA

Fernando Gerchman

Brazil

Berenice Gitomer

USA

Despoina Gkentzi

Greece

Glenda Gobe

Australia

Margarethe Goetz

USA

Sarah Goff

USA

Paraskevi Goggolidou

UK

David Goldfarb

USA

Matthew Griffin

Ireland

Konstadina Griva

Singapore

Jaap Groothoff

Netherlands

Fabrizio Grosjean

Italy

Jakob Gubensek

Slovenia 
Encarna Guillen-Navarro

Spain

Zhiyong Guo

China

Orlando Gutierrez

USA

Mark Guy

UK

Andrew Hall

Switzerland

Bruce Hall

Australia

Garry Handelman

USA

Lisa Harrison-Bernard

USA

Donna H Harward

USA

Junichiro Hayano

Japan

John He

USA

Meian He

China

Martinez Ramirez Hector Ramon

Mexico

Hiddo Heerspink

Netherlands

Debra Higgins

Ireland

Nathan Hill

UK

Ewout Hoorn

Netherlands

Jennifer Hoponick Redmon

USA

Sabine Horn

Austria

Masato Hoshi

USA

Seyed Mohammadmehdi Hosseini

Moghaddam

Iran

Raymond Hsu

USA
Weixin Hu

China

Shih-Han Huang

Canada

Michael Hultström

Sweden

Masimango Imani Mannix

Congo, Democratic Republic

Yoshitaka Isaka

Japan

Bernard Jaar

USA

Tazeen Jafar

Singapore

Deepika Jain

USA

Navin Jaipaul

USA

Matthew James

Canada

Aleksandar Jankovic

Serbia

Faical Jarraya

Tunisia

Vanita Jassal

Canada

Guillaume Jean

France

Robert Jenkins

UK

Julia S. Johansen

Denmark

Kirsten Johansen

USA

Heather Johnson

USA

Harald Jorstad

Netherlands

Veena Joshi

Singapore

Stephen Juraschek

USA

Claudine Jurkovitz

USA
Sahir Kalim

USA

Tomohiro Kamoda

Japan

Houda Kanoun

Tunisia

Vikas Kapil

UK

Andre Kaplan

USA

Ameet Karambelkar

USA

Riyaj Kasekar

USA

Mitsuhiro Kawano

Japan

Frieder Keller

Germany

Jessica Kendrick

USA

Eric Kerns

USA

Zeid Khitan

USA

In Joo Kim

South Korea

Won Kim

Korea, South

Seoyoung Kim

USA

Dimitrios Kiortsis

Greece

Alexander Kirsch

Austria

Krzysztof Kiryluk

USA

Hiroyuki Kobori

USA

Hirotaka Komaba

Japan

Judith Kooiman

Netherlands

Jeroen Kooman

Netherlands 
Marion Koopman

Netherlands

Jay Koyner

USA

Andrea Kramer

Netherlands

Kalimuthu Krishnamoorthy

USA

Mei-Chuan Kuo

Taiwan

Ira Kurtz

USA

Mamoru Kusaka

Japan

Mi-Kyoung Kwak

Korea, South

Arjan Kwakernaak

Netherlands

Soon Hyo Kwon

Korea, South

Jean-Philippe Lafrance

Canada

Quirino Lai

Italy

Vijay Lapsia

USA

Thomas Larsen

USA

Alexandre Lautrette

France

Maurice Laville

France

Athina Lavrentieva

Greece

David Leaf

USA

Nelson Leung

USA

Moshe Levi

USA

Keith Levine

USA

Joshua Lewis

Australia
Karl Lhotta

Austria

Luyuan Li

China

Sophie Liabeuf

France

John Lieske

USA

Monica Limardo

Italy

Wender Lin

Taiwan

Yen-Chung Lin

Taiwan

Gregor Lindner

Switzerland

Kasia Lipska

USA

Francesco Locatelli

Italy

Carl Lombard

South Africa

David Long

UK

Joseph Longenekcer

Kuwait

Giuseppe Lucarelli

Italy

Giancarlo Lucchetti

Brazil

Randy Luciano

USA

Antonio Lupo

Italy

Francisco Maduell

Spain

Mitra Mahdavi-Mazdeh

Iran

Cherry Mammen

Canada

Filippo Mangione

Italy

Maria Martinez Cantarin

USA
Bashir Matata

UK

Vladimir Matchkov

Denmark

Sandro Mazzaferro

Italy

Marilda Mazzali

Brazil

William Mccumbee

USA

D. Olga Mcdaniel

USA

Susan Mclennan

Australia

Anita Mehrotra

USA

Kathan Mehta

USA

Bjorn Meijers

Belgium

Michal Melamed

USA

Cathy Mendelsohn

USA

David Mendelssohn

Canada

Shanthi Mendis

Switzerland

Robert Menzies

UK

Piergiorgio Messa

Italy

Wieneke Michels

USA

Farzanehsadat Minoo

Iran

Roberto Minutolo

Italy

Dana Miskulin

USA

Brett Mitchell

USA

Sandip Mitra

UK 
Orson Moe

USA

Marcus Moeller

Germany

Femke Mollema

Netherlands

Stig Mølsted

Denmark

Divya Monga

USA

Alessandro Montanelli Italy

Rafique Moosa

South Africa

Olivier Moranne

France

Rosa Moyses

Brazil

Miguel Moyses Neto

Brazil

Shrikant Mulay

Germany

Luisa Murer

Italy

Corrado Murtas

Italy

Raghavan Murugan

USA

Micho Nagata

Japan

Farid Nakhoul

Israel

Sankar Navaneethan

USA

Ana Navas-Acien

USA

Guy Neild

UK

Craig Nelson

Australia

Giuseppe Stefano Netti Italy

Hannes Neuwirt

Austria
David Nikolic-Paterson

Australia

Shinichi Nishi

Japan

Claudia Nold

Australia

Donal O'Donoghue

UK

Aghogho Odudu

UK

William Oetting

USA

Ann O'Hare

USA

Yasushi Ohashi

Japan

Ercan Ok

Turkey

Klaus Olgaard

Denmark

Alberto Ortiz

Spain

Alfonso Otero Gonzalez

Spain

Zeynep Birsin Özçakar

Turkey

Carlos Palant

USA

Antonello Pani

Italy

Vincenzo Panichi

Italy

Rulan Parekh

Canada

Jung Tak Park

Korea, South

Andreas Pasch

Switzerland

Thakor Patel

USA

Rachel Patzer

USA

Adriano Peris

Italy
Maria Picken

USA

Francesca Pistoia

Italy

Laura Plantinga

USA

Carlos Poli De Figueiredo

Brazil

Rafael Ponikvar

Slovenia

Roberto Pontremoli

Italy

Stefan Porubsky

Germany

Maurizio Postorino

Italy

Valentina Postorino

Italy

Hans Pottel

Belgium

Giuseppe Quintaliani

Italy

Wajeh Qunibi

USA

Jochen Raimann

USA

Luca Rampoldi

Italy

Panduranga Rao

USA

Hugh Rayner

UK

Elrashdy Redwan

Saudi Arabia

Kevin Regner

USA

Flavio Reis

Portugal

Kyle Richards

USA

Azra Rizwan

Pakistan

Michael Robson

UK 
Dario Roccatello

Italy

Alexander Rosenkranz

Austria

Mitchell Rosner

USA

Michael Ross

USA

Dvora Rubinger

Israel

Rupam Ruchi

USA

Gill Rumsby

UK

Domenico Russo

Italy

Abraham Rutgers

Netherlands

Dong-Ryeol Ryu

Korea, South

Ernesto Sabath

Mexico

Takako Saeki

Japan

Marcus Saemann

Austria

Seyed Ehsan Saffari

Iran

Amrik Sahota

USA

Hiroyuki Sakurai

Japan

Fadi Salem

USA

Richard Sandford

UK

Rajiv Saran

USA

Patrick Saudan

Switzerland

Judy Savige

Australia

John Sayer

UK
Arzu Sayiner

Turkey

Francesco Paolo Schena

Italy

Rebecca Scherzer

USA

Gernot Schilcher

Austria

Georg Schlieper

Germany

Ute Scholl

Germany

Savino Sciascia

Italy

Antonio Seguro

Brazil

Nicholas Selby

UK

David Selewski

USA

Donal Sexton

Ireland

Tariq Shafi

USA

Sanjeev Shah

USA

Linda Shavit

Israel

Neil Sheerin

UK

Kanji Shishido

Japan

Sandra Silveiro

Brazil

Justin Silver

Israel

Ana Cristina Simoes E Silva

Brazil

Pravin Singhal

USA

Ranga Siriwardhana

Sri Lanka

Meghan Sise

USA
Itzchak Slotki

Israel

Jayne Smith-Palmer

Switzerland

Laura Smyth

UK

Anna Solini

Italy

Ronak Soni

India

Manish Sood

Canada

Clemente Sousa

Portugal

Kevin Sowinski

USA

Stephen Sozio

USA

Adrian Specogna

Canada

Marijn Speeckaert

Belgium

J David Spence

Canada

Bruce Spinowitz

USA

Austin Stack

Ireland

Constantinos J Stefanidis

Greece

Tomasz Stompor

Poland

Elani Streja

USA

Toshihiro Sugiura

Japan

Ernest Sumaili

Congo, Democratic Republic

Randall Sung

USA

Chanderdeep Tandon

India

Sydney C.W. Tang

Hong Kong 
Adis Tasanarong

Thailand

Avelino Teixeira

USA

Karthik Tennankore

Canada

Charuhas Thakar

USA

Mae Thamer

USA

Nicola Thomas

UK

Charlotte Thomas-Hawkins

USA

Joji Tokita

USA

Ashita Tolwani

USA

Allison Tong

Australia

Massimo Torreggiani

Italy

Yusuke Tsukamoto

Japan

J. Kevin Tucker

USA

Kriang Tungsanga

Thailand

Delphine Tuot

USA

Shigehiko Uchino

Japan

Susumu Uda

Japan

Jaime Uribarri

USA

Johannes G. Van Der Hoeven

Netherlands

Karlijn Van Der Pant

Netherlands
Frank Van Der Sande

Netherlands

Harry Van Goor

Netherlands

Barbara Van Munster

Netherlands

Mariacristina Vecchio

Italy

Andrea Veltri

Italy

Francisco Veronese

Brazil

Rachel Vieux

France

Giuseppe Villa

Italy

Folkert Visser

Netherlands

Astrid Wahl

Norway

Ron Wald

Canada

Stephen Waldek

UK

Rebecca Walker

UK

Stephen Walsh

UK

Yin Wang

USA

Makoto Watanabe Japan

Amy Waterman

USA

Steven Weisbord

USA

Catharina Wesseling

Finland

Katherine Wesseling-Perry USA
Francis Wilson

USA

Dawn Wolfgram

USA

Keng Thye Woo

Singapore

Alan Wright

UK

Julie Wright-Nunes

USA

Christina Wyatt

USA

Gaoqiang Xie

China

Eishin Yaoita

Japan

Pia Yngman-Uhlin

Sweden

Eric Young

USA

Sue Yung

China

Stuart Yuspa

USA

Hong Zhang

China

Ying Zhang

USA

Carmine Zoccali

Italy

Li Zuo

China

Received: 5 January 2015 Accepted: 5 January 2015

Published: 23 January 2015

doi:10.1186/1471-2369-16-1

Cite this article as: Henderson: BMC

Nephrology reviewer acknowledgement

2014. BMC Nephrology 2015 16:1. 